Supporting Information for

\title{
Electrostatic Assembly of a Titanium Dioxide@Hydrophilic Poly(phenylene sulfide) Porous Membrane with Enhanced Wetting Selectivity for Separation of Strongly Corrosive Oil- water Emulsions
}

Na Han", 1, a, b, c, Chao Yang', a, b, d, Zongxuan Zhang,

b, Changye Han ${ }^{\mathrm{a}, \mathrm{b}}$, Zhenyu Cuia, b, Wei Li ${ }^{\mathrm{a}, \mathrm{b}}$, Xingxiang Zhang*a, b

a State Key Laboratory of Separation Membranes and Membrane Processes, Tianjin 300387, China.

b School of Materials Science and Engineering, Tianjin Polytechnic University, Tianjin 300387, China.

c Textile Engineering, Chemistry and Science Department, North Carolina State University, Raleigh, NC, 27606, United States.

${ }^{\mathrm{d}}$ Key Laboratory for Green Chemical Technology of Ministry of Education, School of Chemical Engineering and Technology, Tianjin University, Tianjin 300072, China.

${ }^{*}$ Corresponding author.

${ }^{1}$ These authors contributed equally to this work.

E-mail address: nhan@ncsu.edu; zhangpolyu@aliyun.com 


\section{Preparation of the PPS microporous membrane.}

The PPS microporous membrane was prepared by the homemade lab device shown in Figure S1(a) via the thermally induced phase separation process (TIPS). The mixture of PPS, CPL, and DBS with the mass ratio of 25:60:15 was put into a three-neck flask, stirred, and heated to $255^{\circ} \mathrm{C}$ for 30 minutes in a nitrogen atmosphere. After degassing for $10 \mathrm{~min}$, the as-prepared solution was quickly poured onto the mold to form a membrane. After cooling the mold in the air, the solidified membrane was immersed in absolute ethyl ethanol for $48 \mathrm{~h}$ and then in pure water for another $24 \mathrm{~h}$. The final membrane was dried at $50{ }^{\circ} \mathrm{C}$ for $24 \mathrm{~h}$.

(a)

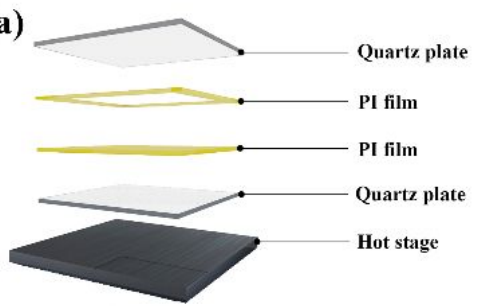

(b)

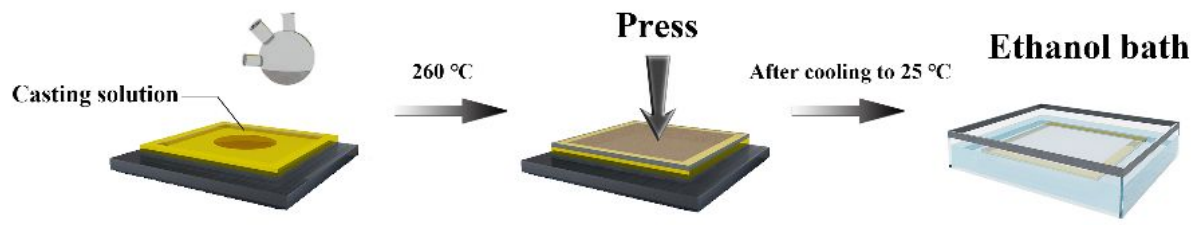

Figure S1. a, Homemade lab device for the preparation of PPS microporous membranes via TIPS. b, Schematic diagram of the preparation of PPS microporous membranes via TIPS. 


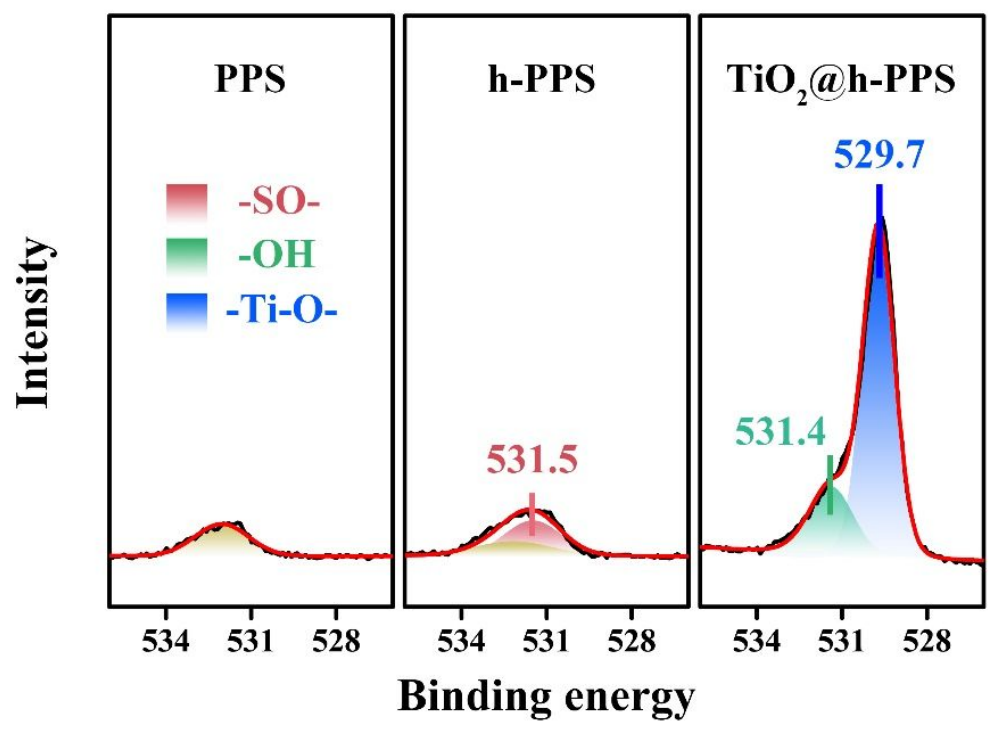

Figure S2. O 1s high-resolution spectra and corresponding XPS data of the PPS, h-PPS, and $\mathrm{TiO}_{2} @$ h-PPS membranes.

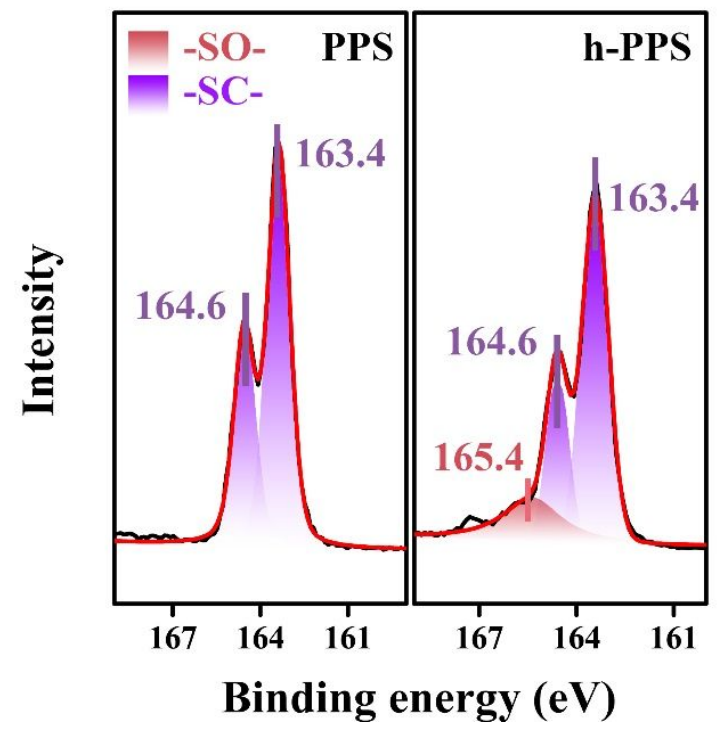

Figure S3. S 2p high-resolution spectra and corresponding XPS data of the PPS and h-PPS membranes. 

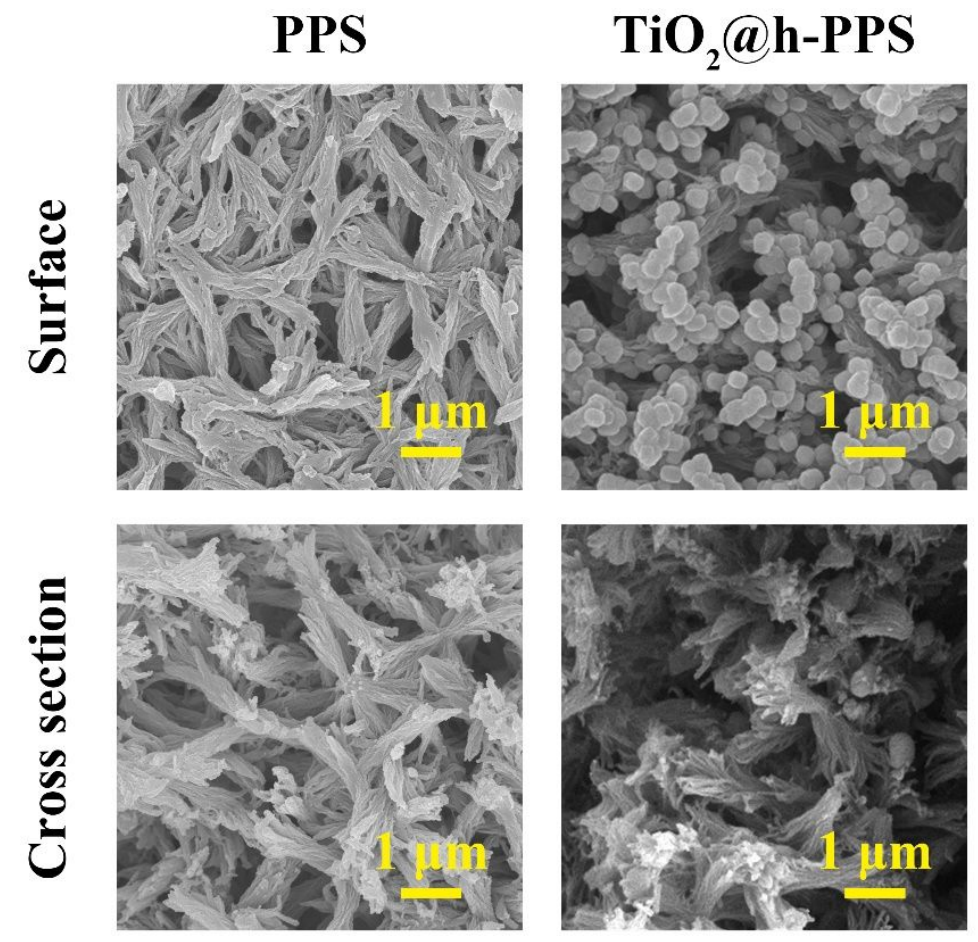

Figure S4. SEM images of the PPS and $\mathrm{TiO}_{2} @$ h-PPS membranes.

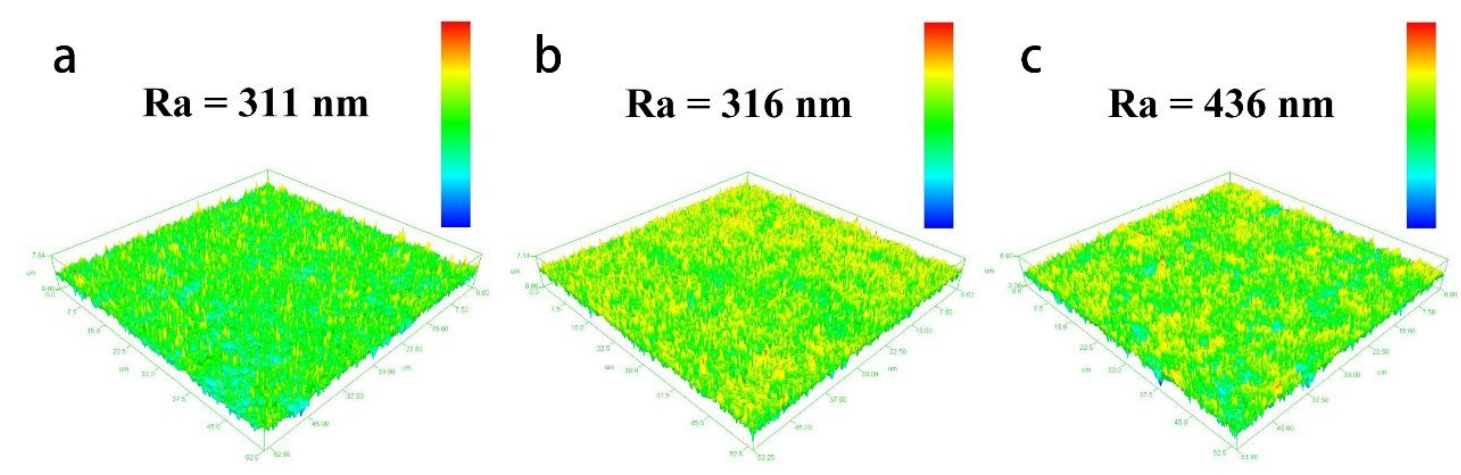

Figure S5. Scanning confocal microscope images and Ra data of the (a) PPS, (b) h-PPS, and (c) $\mathrm{TiO}_{2} @$ h-PPS membranes 

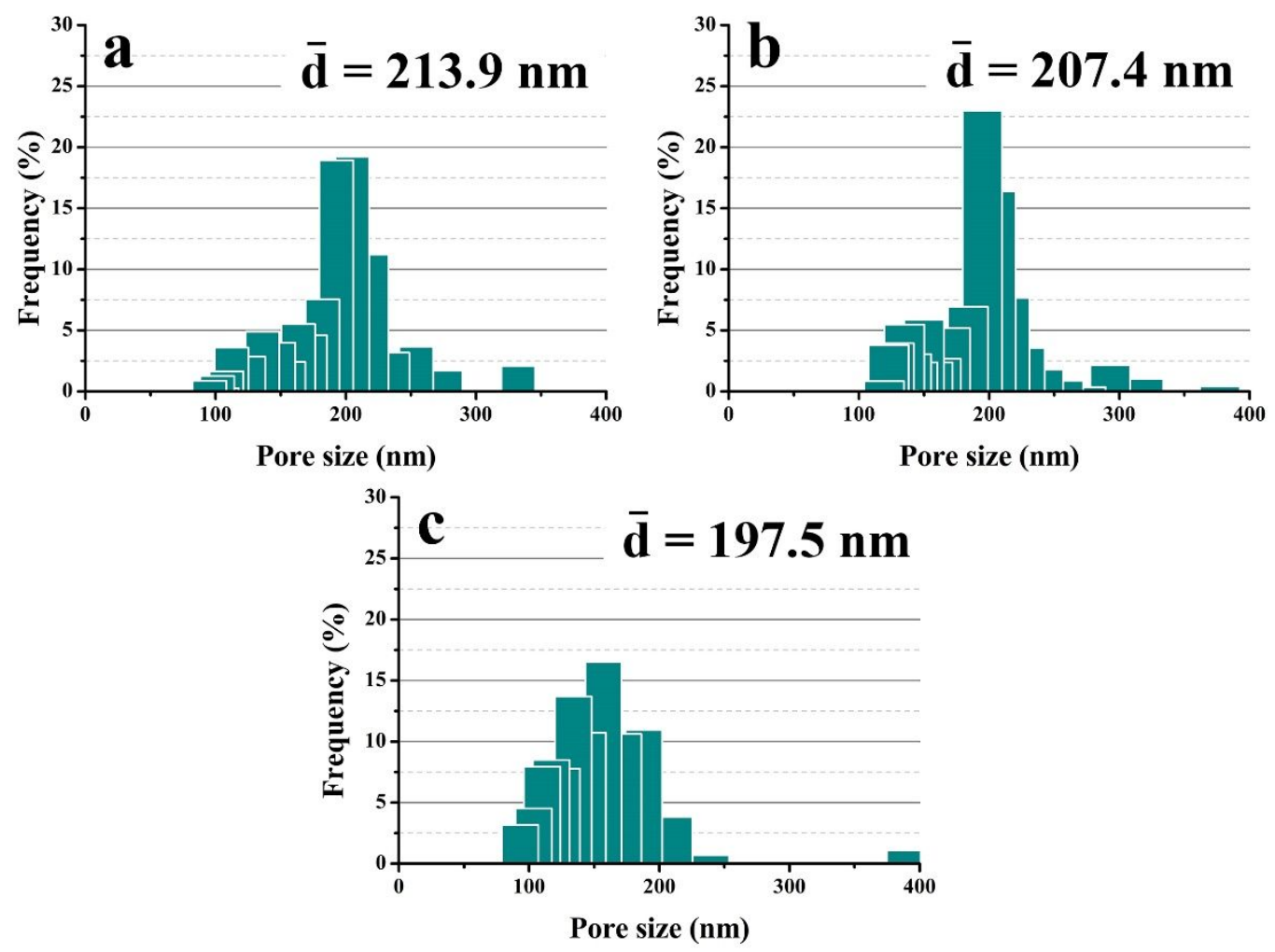

Figure S6. Pore size distributions of the (a) PPS, (b) h-PPS, and (c) TiO $\mathrm{T}_{2}$ ah-PPS membranes.

The pore size distributions of the PPS, h-PPS, and $\mathrm{TiO}_{2} @$ ah-PPS membranes are presented in Figure S6. The average pore diameters of the PPS, h-PPS, and TiO $\mathrm{O}_{2} @$ h-PPS membranes are $213.9 \mathrm{~nm}, 207.4 \mathrm{~nm}$, and $197.5 \mathrm{~nm}$, respectively. This value of the $\mathrm{TiO}_{2} @$ h-PPS membrane shows a little or slight change compared to those of the PPS and h-PPS membranes due to the growth of $\mathrm{TiO}_{2}$ on the membrane. 

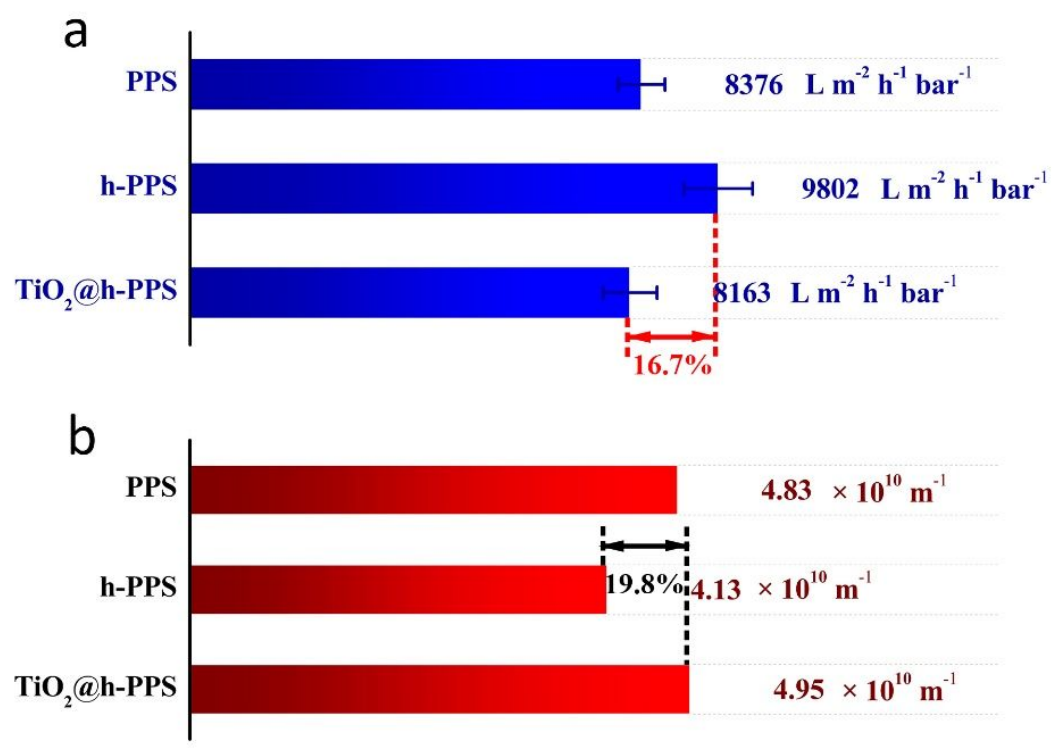

Figure S7. a, Pure water fluxes and b, transmembrane resistance of the PPS, h-PPS, and $\mathrm{TiO}_{2} @$ h-PPS membranes.

Pure water fluxes are evaluated via a dead-end filtration, and the value is obtained by Equation (S1):

Pure water flux $=\frac{V}{A \times t}$

Where $V, A$, and $t$ denote the volume of the water $(\mathrm{L})$, the effective membrane area $\left(\mathrm{m}^{2}\right)$, and the running time (h), respectively. As shown in Figure S6, the fluxes of the PPS, hPPS, and $\mathrm{TiO}_{2} @$ h-PPS membranes are $8376 \mathrm{~L} \mathrm{~m}^{-2} \mathrm{~h}^{-1}$ bar $^{-1}, 9802 \mathrm{~L} \mathrm{~m}^{-2} \mathrm{~h}^{-1}$ bar $^{-1}$, and 8163 $\mathrm{L} \mathrm{m}^{-2} \mathrm{~h}^{-1}$ bar-1, $^{-1}$ respectively. This value of the h-PPS membrane is higher than that of the PPS membrane, resulting from the improvement of hydrophilcity. For the $\mathrm{TiO}_{2} @ \mathrm{~h}-\mathrm{PPS}$ membrane, the flux only shows slight decrease after the growth of $\mathrm{TiO}_{2}$. This limited 
decline is beneficial to maintain the high flux of the h-PPS membrane, achieving surface decoration and performance optimization without dramatically increasing transmembrane resistance.

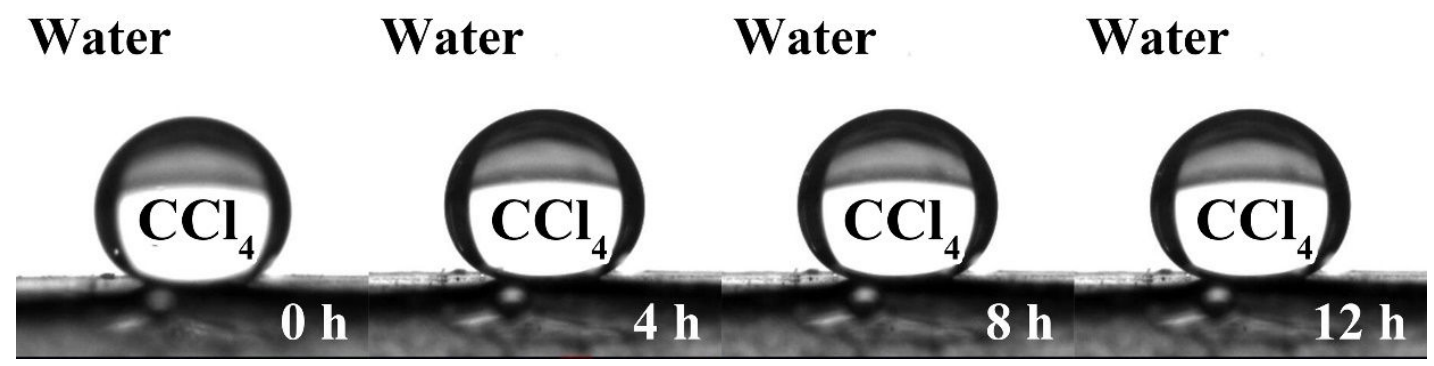

Figure S8. Long-term observation of underwater OCA on the $\mathrm{TiO}_{2} @$ h-PPS membrane.

The underwater OCA shows little change within $12 \mathrm{~h}$, demonstrating that the underwater OCA is robust on the TiO $\mathrm{Ti}_{2}$ h-PPS membrane.

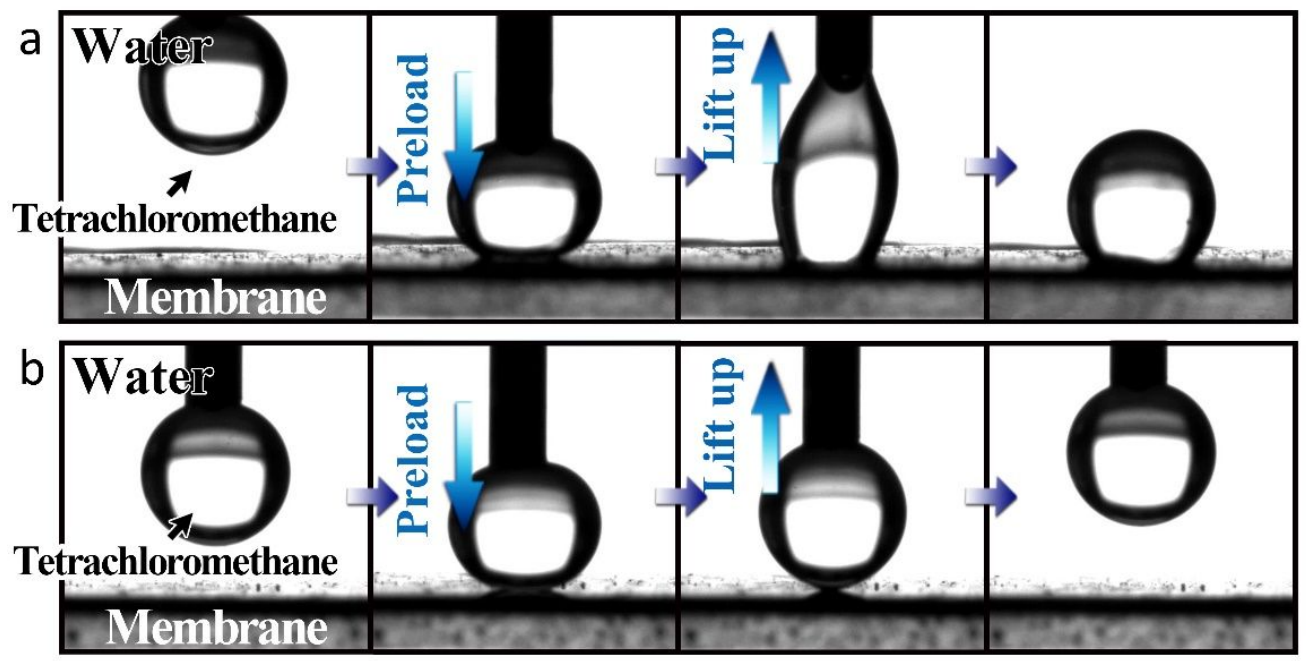

Figure S9. Dynamic oil adhesion test of the h-PPS and $\mathrm{TiO}_{2} @$ h-PPS membranes. The oil droplets are tetrachloromethane. 
Tetrachloromethane droplets $(5 \mu \mathrm{L})$ are placed and preloads on the surface of the h-PPS and $\mathrm{TiO}_{2} @$ h-PPS membrane under water, respectively. Then, the droplets are lifted up slowly. During the lifting process, the droplet on the $\mathrm{TiO}_{2} @$ h-PPS membrane shows almost on deformation and detaches from the membrane surface completely. While, the droplet on the h-PPS membrane is elongated after being lifted up and adhered to the membrane. These phenomena illustrate that ultralow adhesion achieve after coating the $\mathrm{TiO}_{2}$ layer on the h-PPS membrane surface.

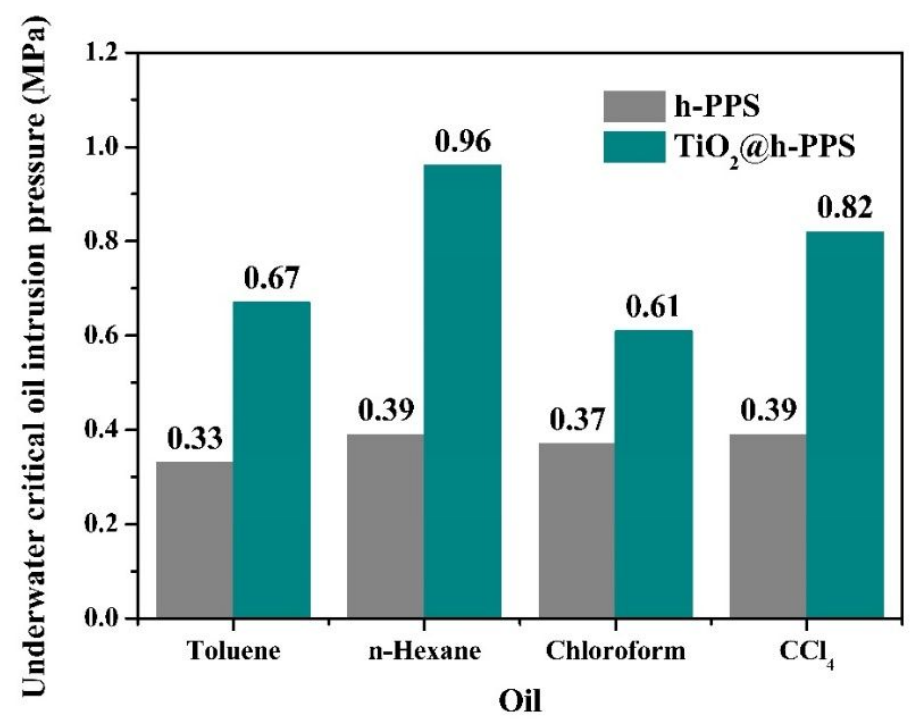

Figure S10. Underwater critical oil intrusion pressures of the h-PPS and $\mathrm{TiO}_{2} @$ h-PPS membranes. 


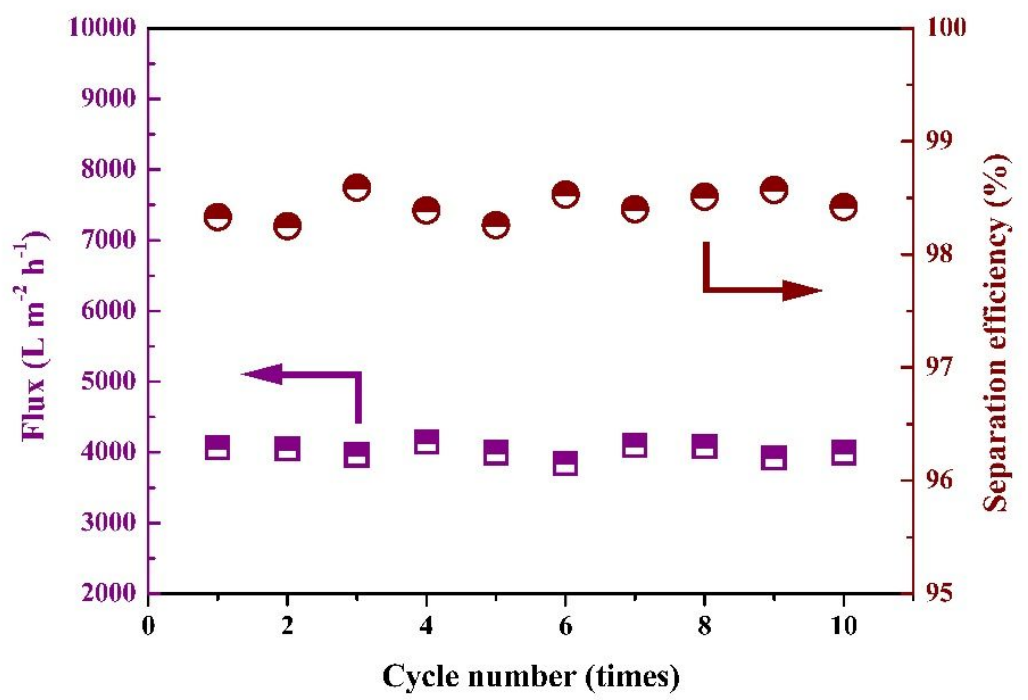

Figure S11. Cycling performance of the $\mathrm{TiO}_{2} @$ h-PPS membrane for the separation of the surfactant-stabilized T/W emulsion.

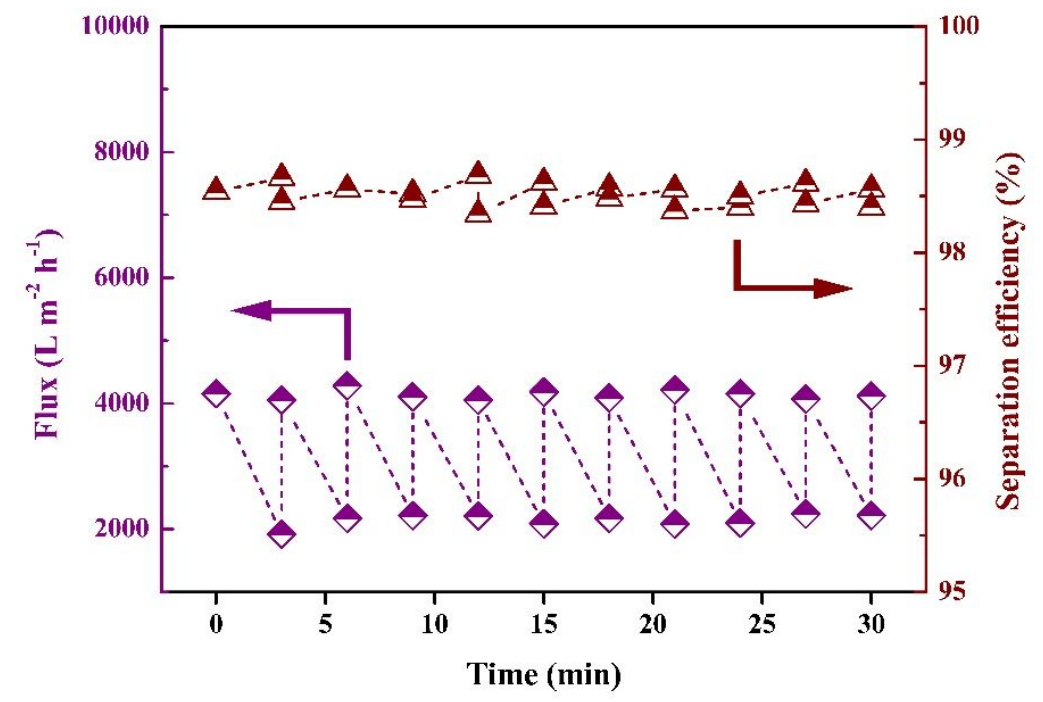

Figure S12. Separation performances of the $\mathrm{TiO}_{2} @$ h-PPS membrane varied with the separation time for the separation of the surfactant-stabilized T/W emulsion. 